\title{
Current Approaches to Improve Balance in Down Syndrome Population-A Systematic Review
}

\author{
Nazia Adeeb ${ }^{1, *}$, Sumaira Imran Farooqui ${ }^{2}$, Abid Khan ${ }^{2}$, Jaza Rizvi ${ }^{2}$ and Syed Abid Mehdi \\ $\mathrm{Kazmi}^{3}$
}

${ }^{1}$ A.C.E.L.P (Institute of Child Development), 197/8, Rafiqui Shaheed Road, Opposite Jinnah Postgraduate Medical Centre, Karachi, Pakistan

${ }^{2}$ Ziauddin College of Rehabilitation Sciences, Ziauddin University, Plot \# E - 40/1- Wahid Colony Block - B North Nazimabad, Karachi, Pakistan

${ }^{3}$ Dr. Ziauddin Hospital, 4/B Shahrah-e-Ghalib Rd, Block 6 Clifton, , Sindh 75600, Karachi, Pakistan

\begin{abstract}
Down syndrome is one of the most common chromosomal disorders in pediatrics characterized by variable intellectual disability, generalized joint laxity, and hypotonia that compromises their function and causes a delay in developing gross motor skills, poor balance, and coordination. Thus, this study aims to determine the current yet effective treatment approaches to improve balance in the Down syndrome population. The studies were explored across seven electronic databases that include MEDLINE, PubMed, Cochrane Library, Google Scholar, Scopus, PEDro, and Web of Science from inception till October 2020 comprised of experimental studies published in English language investigating the effects on balance in children and adults diagnosed with DS considering different interventions. A total of 1,570 records were retrieved from seven electronic databases published between the year's tenure of 2013-2020. 144 full-text papers were extracted to be reviewed, of which only 18 experimental studies were selected on the basis of inclusion criteria that involved 493 Down syndrome patients, investigated the effects of therapeutic exercises, manual therapy techniques, and patient-related instructions on standardized balance scales/tests. It was concluded that all the included trials demonstrated significantly profound effects in improving the static and dynamic balance of Down syndrome patients. Therefore, none of the interventions is declared as superior to another in terms of obtained results. Furthermore, these diverse interventions need to be investigated more for better understating and generalizability of outcomes.
\end{abstract}

Keywords: Down syndrome, Trisomy 21, Therapy, Proprioception, Equilibrium, Position Sense.

\section{INTRODUCTION}

Down Syndrome (DS), a subcategory of intellectual disability, is one of the most common chromosomal disorders in the pediatric population caused by full trisomy $21(94 \%)$, mosaicism (2.4\%), or translocations $(3.3 \%)$ [1]. World Health Organization (WHO) documents the global incidence of children with Down Syndrome to be 1 out of $600-1000$ live births [2]. The condition is characterized primarily by variable intellectual disability and peculiar physical features like distinct facial phenotype, short stature, and generalized joint laxity, and hypotonia with effect on health and development [3, 4]. Hypotonicity causes decreased muscular strength and activity tolerance and hypermobility in joints, compromising their function and delay in developing gross motor skills, poor balance, and coordination [1, 5, 6]. Evidence shows that motor development in this population has a different profile than that of a child without disabilities because the age category at which a specific motor level is reached is higher [7]. Besides, DS children may exhibit a wide

*Address correspondence to this author at the A.C.E.L.P (Institute of Child Development). 197/8, Rafiqui Shaheed Road, Opposite Jinnah Postgraduate Medical Centre, Karachi, Pakistan; Tel: 021-3567557;

E-mail: nadeeb@hotmail.com range of co-morbidities affecting various systems like respiratory, cardiac, immune, endocrine, etc. [8]. Despite this fact, survival has increased considerably in the last 50 years from $<50 \%$ in the mid-1990s to $95 \%$ in the early 2000 s with a life expectancy of 60 years [9]. One of the reasons for this improvement can be attributed to advancements in medical technology with prenatal detection and early intervention. A paradigm shift is observed in society's attitude towards the normalization of the lives of people with DS [8]. This has led to a better state of health, a higher degree of autonomy, integration, and inclusion of this population in the community during the last two decades [8]. Despite all these advances, the incidence has also grown in the last decades, drawing the attention of scientists from various fields on its causes, forms of manifestation, and ways of treating the children diagnosed with this syndrome with the development of a comprehensive therapeutic approach [7].

Multiple studies suggested that DS children present with decreased balance, affecting motor skill proficiency and physical activity. Therefore, approaches that deal with this issue are of utmost importance as their participation in various walks of life is increasing as well as in athletic and sports events 
$[10,11]$. Moreover, improved gross motor skills and balances would help in increasing their role in life activities and enhancing their self-esteem [12]. Experimental Studies for Down syndrome to improve balance are scarce, creating a vacuum in this domain [8]. Thereby arising a need to explore various therapeutic approaches to ameliorate balance among the population [13].

Furthermore, to the best of author's knowledge, no such review that highlights various modes of intervention to improve balance is found. Therefore, this review intends to synthesize various effective and efficient methods that enhance balance in the DS population. Moreover, disseminate awareness amongst the rehab professionals regarding these methods to enhance their knowledge of diverse forms of intervention available and increase the horizon of the specialist, especially physical therapist, to improvise the plan of care. Thus, the identification of effective modes of intervention would enhance and upgrade therapists' skills, which will eventually benefit the Down syndrome population specifically and society in general. Therefore, the objective of this systematic review is to evaluate the current strategies to improve balance in the DS population, as well as to identify the most effective amongst them.

\section{METHODS}

This study followed Preferred Items for Systematic Reviews and Meta-Analysis (PRISMA) guidelines to ensure that the pertinent evidence was sourced and synthesized into the conscripting of this commentary.

\section{Databases and Literature Surveillance}

The studies were explored through a systematic approach across seven electronic databases: MEDLINE, PubMed, Cochrane Library, Google Scholar, Scopus, PEDro, and Web of Science from inception until October 2020. Filters about publication dates or language were applied. The authors used Medical Subject Headings (MeSH) to identify the related entry terminology in our searches comprised of "Balance AND Down Syndrome", "Interventions AND Balance AND Down Syndrome", "Balance AND Down Syndrome: RCTs".

\section{Inclusion Criteria}

(1) Participants were children and adults diagnosed with DS, (2) Experimental studies, (3) Intervention constitutes of therapeutic exercise, manual therapy techniques, patient-related instructions, and orthotic devices, (4) Outcome measure; balance and standardized balance scale/test, (5) Studies published during 2013-2020, (6) Preferred language; English.

\section{Exclusion Criteria}

Data reported does not address the Down syndrome interventions searched, (2) Data reported was unable to analyze for balance specifically, (3) Literature preceding 2013, (4) Unavailable full-texts (5) Non-English studies (6) Duplication.

\section{Study Selection}

Literature Search was performed by the first author, and screening was executed according to the aforementioned eligibility criteria by reviewing titles. Furthermore, advice was sought from experienced reviewers for any disparity on inclusion/exclusion of studies reaching a final list of studies.

\section{Data Extraction}

The extraction of data was done by two researchers in a systematic way from the study list finalized by two independent reviewers. The extracted data included information on (1) Author and publication year (2) Characteristics of the participants (Number of Participants, Age, Gender, Severity of ID) (3) Intervention (Frequency, Duration) (4) Outcome Measures (5) Summary of results.

\section{Risk of Bias}

The risk of bias was evaluated by using the Cochrane tool for assessing the risk of bias [14] in random allocation, allocation concealment, blinding of participants and outcome assessment, incomplete outcome data, selective reporting, and other biases.

\section{RESULTS}

\section{Selection and Characteristics of Studies}

A total of 1,570 records were retrieved from seven electronic databases comprised of MEDLINE, PubMed, Cochrane Library, Google Scholar, Scopus, PEDro, and Web of Science published between 2013-2020. After the initial screening, 870 records were excluded due to unmatched title and abstract, whereas 556 removed due to duplications, irrelevant and nonEnglish language. Further, 144 full-text papers were extracted to be reviewed, of which only 18 


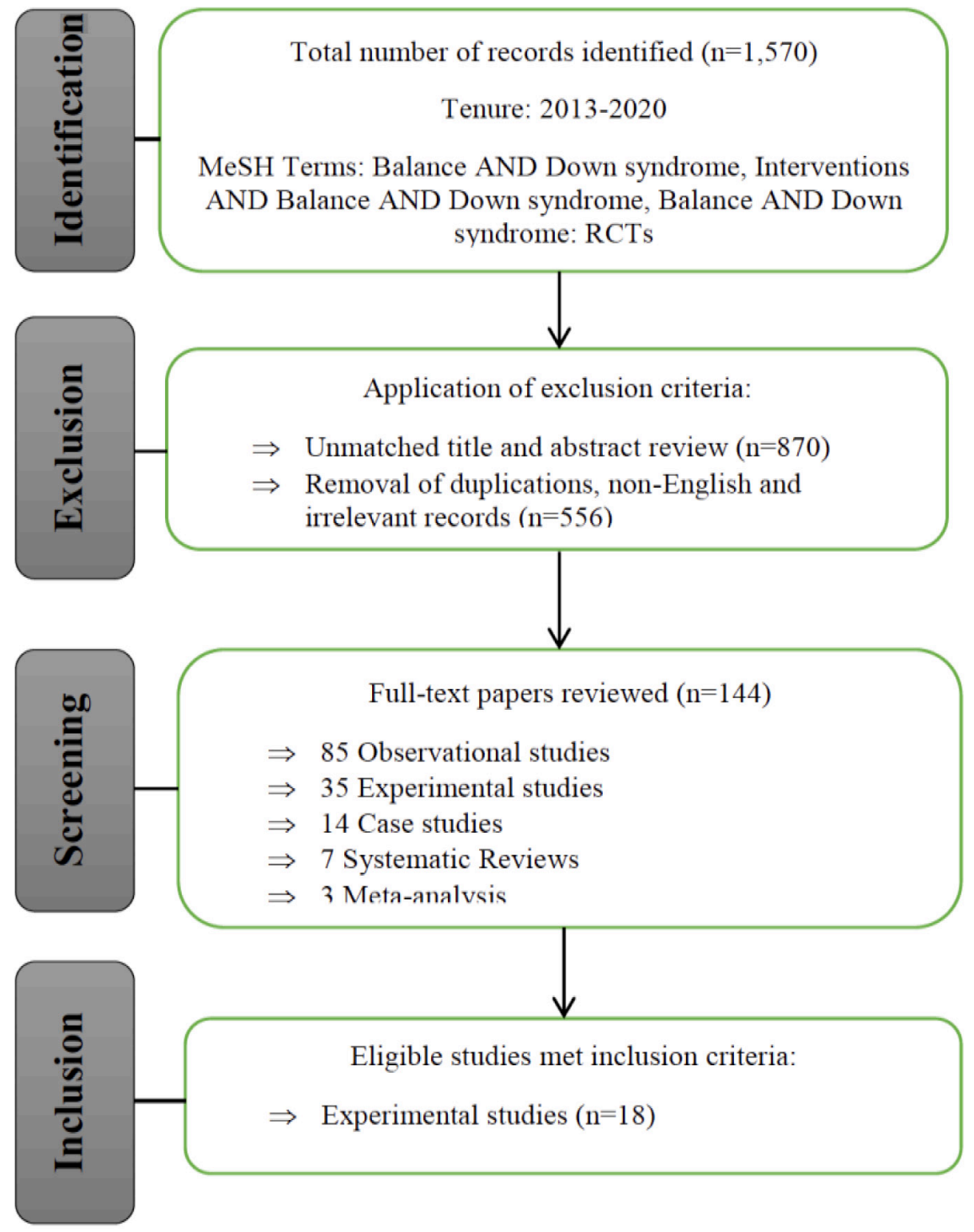

Figure 1: Represents the conceptualization of the review process.

experimental studies were selected on the basis of inclusion criteria that involved 493 Down syndrome patients, investigated the effects of therapeutic exercises, manual therapy techniques, patient-related instructions, and orthotic devices on standardized balance scales/tests.

The selection of criteria of studies was accomplished in the following subsequent phases, as shown in Figure 1.

\section{Synthesized Findings}

Among the eighteen experimental studies, fourteen studies were Randomized Controlled Trials; three were Quasi-experimental, while only one was Nonrandomized controlled trial. A study conducted by Alsakhawi et al. [15], Eid et al. [20], Ghafar et al. [21], and Reis et al. [22] investigated the effects of traditional/conventional physiotherapy with an adjunct intervention such as treadmill, core stability, isokinetic training, and virtual reality, and the investigation revealed that conventional therapy in combination with an adjunct intervention was more effective than solitary to improve balance among DS patients. Gheitasi et al. [16] and Ghaeeni et al. [26] showed the efficacy of core stability exercises, either alone or in combination with the swiss ball or resistance training, in improving static balance. Similar outcomes were reported by Boer et al. [17] and Hamed et al. [23] regarding aquatic therapy alone or in combination to balance exercises. Further, Eid [25] and Villarroya et al. [28] demonstrated comparable findings with whole-body vibration. Likewise, Kamatchi et al. [10] and Carter et al. [5] exhibited higher effectiveness of vestibular stimulation 
Table 1: Main Characteristics of Participants in the Study $(n=18)$

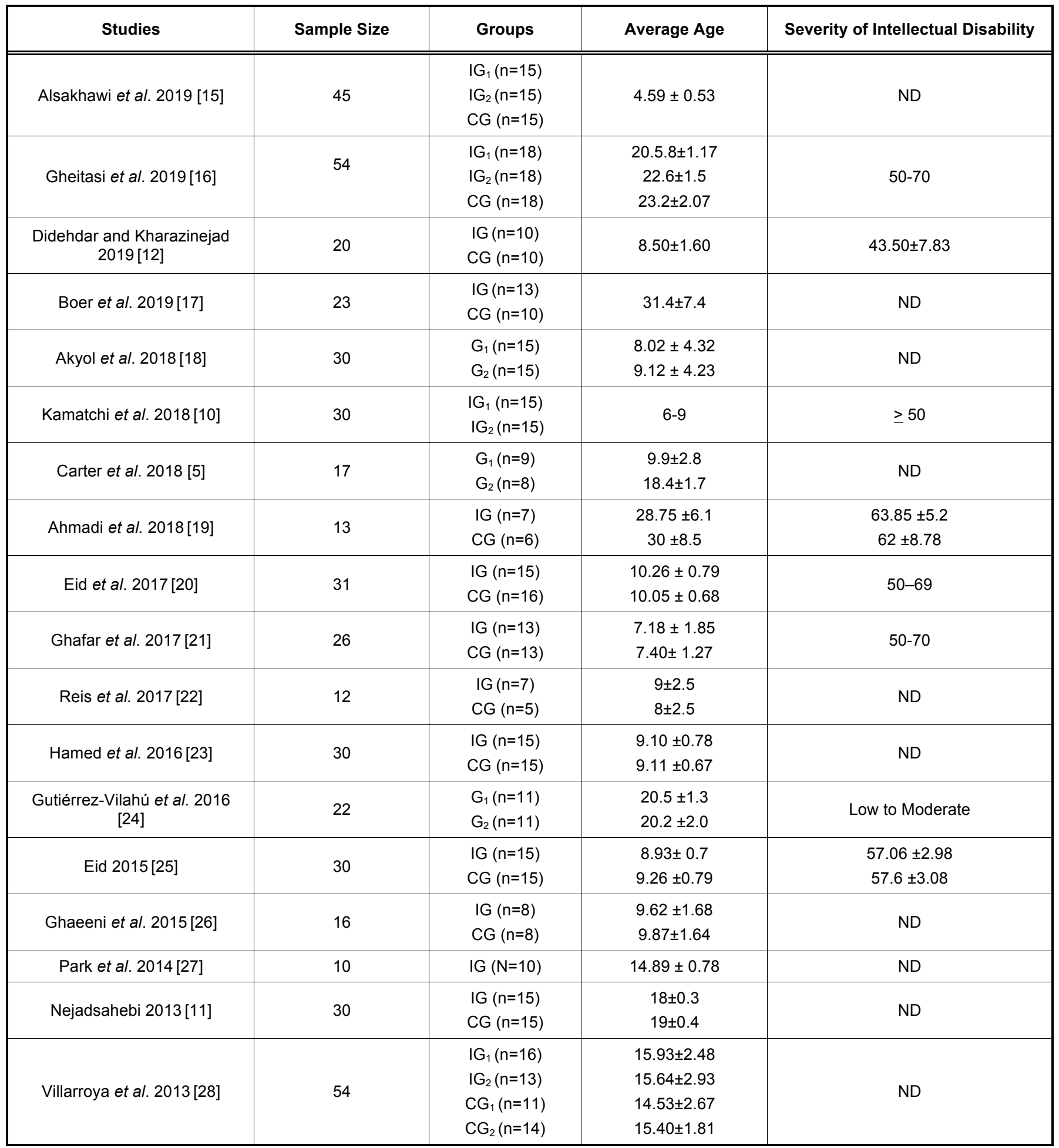

in improving balance. Moreover, Akyol et al. [18] and Ahmadi et al. [19] concluded that functional or gymnastics training with music has led to significant differences in balance. On the other hand, Didehdar and Kharazinejad [12], Park et al. [27], and Nejadsahebi [11] ascertained the significant effects of sensory integration, wobble board training, and rebound therapy, respectively as a single intervention in enhancing static and dynamic balance. However, Gutiérrez-Vilahú et al. [24] indicated that a combination of diverse management through core strength, ballet barre, proprioception, balance exercise, and 
Table 2: Description of the Eligible Studies $(n=18)$

\begin{tabular}{|c|c|c|c|c|c|}
\hline Studies & Study Design & Intervention & Duration & $\begin{array}{l}\text { Measuring } \\
\text { Scale/Test }\end{array}$ & Result \\
\hline Alsakhawi et al. 2019 [15] & $\begin{array}{l}\text { Randomized } \\
\text { Controlled Trial }\end{array}$ & $\begin{array}{c}\mathrm{IG}_{1} \text {-Traditional exercise for } 30 \\
\text { min + treadmill training for } 20 \\
\text { minutes } \\
\mathrm{IG}_{2-\text { Traditional exercise for } 30} \\
\text { min + core stability training for } \\
30 \text { minutes } \\
\text { CG- Traditional exercise for } 60 \\
\text { minutes }\end{array}$ & $\begin{array}{c}\text { 3times/week for } \\
8 \text { weeks }\end{array}$ & $\begin{array}{l}\text { Berg Balance } \\
\text { Scale } \\
\text { Biodex Balance } \\
\text { System }\end{array}$ & $\begin{array}{l}\text { Both IGs } \\
\text { significantly } \\
\text { improved. No } \\
\text { significant } \\
\text { difference } \\
\text { observed } \\
\text { between IGs }\end{array}$ \\
\hline Gheitasi et al. 2019 [16] & $\begin{array}{l}\text { Quasi- } \\
\text { Experimental } \\
\text { Study }\end{array}$ & $\begin{array}{l}I G_{1} \text { Core Stability + Swiss ball } \\
\text { Exercise } \\
I_{2} \text { Core Stability +Total } \\
\text { Resistance Exercise } \\
\text { CG-Daily Routine Activities }\end{array}$ & $\begin{array}{l}3 \text { times/week } \\
\text { for } 8 \text { weeks }\end{array}$ & $\begin{array}{l}\text { Stork Test } \\
\text { Y-Balance }\end{array}$ & $\begin{array}{c}\text { Both IGs } \\
\text { significantly } \\
\text { improved. In } \\
\text { Static Balance, } \\
\text { SBE was better } \\
\text { than TRX while in } \\
\text { Dynamic } \\
\text { Balance, TRX } \\
\text { group was better } \\
\text { than SBE }\end{array}$ \\
\hline $\begin{array}{l}\text { Didehdar and } \\
\text { Kharazinejad' 2019 [12] }\end{array}$ & $\begin{array}{l}\text { Quasi- } \\
\text { Experimental } \\
\text { Study }\end{array}$ & $\begin{array}{l}\text { IG-Sensorimotor Integration } \\
\text { training, } 50 \text { sessions of } 40 \\
\text { minutes } \\
\text { CG-Class Training program }\end{array}$ & 6 times/week & $\begin{array}{l}\text { Modified Stork } \\
\text { Test } \\
\text { Timed Up and } \\
\text { Go Test }\end{array}$ & $\begin{array}{l}\text { IG showed } \\
\text { significant } \\
\text { improvement in } \\
\text { static and } \\
\text { balance }\end{array}$ \\
\hline Boer et al. 2019 [17] & $\begin{array}{l}\text { Non- } \\
\text { randomized } \\
\text { Controlled Trial }\end{array}$ & $\begin{array}{c}\text { IG-Aquatic Training Program for } \\
\text { 35-45 minutes } \\
\text { CG-ND }\end{array}$ & $\begin{array}{l}3 \text { times/week } \\
\text { for } 6 \text { weeks }\end{array}$ & $\begin{array}{l}\text { Standing on One } \\
\text { Leg } \\
\text { 8ft Up and Go } \\
\text { Sit-to-Stand }\end{array}$ & $\begin{array}{l}\text { IG significantly } \\
\text { improved than } \\
\text { CG with strong to } \\
\text { medium effect } \\
\text { sizes }\end{array}$ \\
\hline Akyol et al. 2018 [18] & $\begin{array}{l}\text { Randomized } \\
\text { Controlled Trial }\end{array}$ & $\begin{array}{l}\mathrm{G}_{1}-\mathrm{Gymnastics} \text { Training with } \\
\text { Music. } 1.5-2 \text { hours } \\
\mathrm{G}_{2} \text {-Gymnastics Training with } \\
\text { Music. } 1.5-2 \text { hours }\end{array}$ & $\begin{array}{l}2 \text { times/week } \\
\text { for } 16 \text { weeks }\end{array}$ & $\begin{array}{l}\text { Flamingo } \\
\text { Balance Test } \\
\text { Functional } \\
\text { Reach Test }\end{array}$ & $\begin{array}{l}\text { Significant } \\
\text { differences in } \\
\text { both groups; } \\
\text { however, } \mathrm{G}_{1} \\
\text { improved more } \\
\text { than } \mathrm{G}_{2}\end{array}$ \\
\hline Kamatchi et al. 2018 [10] & $\begin{array}{l}\text { Randomized } \\
\text { Controlled Trial }\end{array}$ & $\begin{array}{l}\mathrm{G}_{1} \text {-Weight-Bearing Exercises } \\
\text { for } 30 \text { minutes } \\
\mathrm{G}_{2} \text {-Vestibular Stimulation for } 30 \\
\text { minutes }\end{array}$ & $\begin{array}{l}7 \text { days/week for } \\
6 \text { weeks }\end{array}$ & $\begin{array}{c}\text { Pediatric } \\
\text { Balance Scale }\end{array}$ & $\begin{array}{l}\mathrm{G}_{2} \text { showed higher } \\
\text { effectiveness in } \\
\text { improving } \\
\text { balance than } \mathrm{G}_{1}\end{array}$ \\
\hline Carter et al. 2018 [5] & $\begin{array}{l}\text { Randomized } \\
\text { Controlled Trial }\end{array}$ & $\begin{array}{c}\mathrm{G}_{1} \text { and } \mathrm{G}_{2} \text { received Vestibular } \\
\text { Stimulation Exercise for } 10 \\
\text { mins }\end{array}$ & $\begin{array}{l}2 \text { times/week } \\
\text { for } 16 \text { weeks }\end{array}$ & $\begin{array}{l}\text { Bruininks } \\
\text { Oseretsky Test } \\
\text { of Motor } \\
\text { Proficiency }\end{array}$ & $\begin{array}{c}\text { Balance } \\
\text { improved more in } \\
\mathrm{G}_{2} \text { than } \mathrm{G}_{1}\end{array}$ \\
\hline Ahmadi et al. 2018 [19] & $\begin{array}{l}\text { Randomized } \\
\text { Controlled Trial }\end{array}$ & $\begin{array}{l}\text { IG -Functional Strength Training } \\
\text { for } 60 \text { mins } \\
\text { CG-Usual activities }\end{array}$ & $\begin{array}{l}3 \text { times/week } \\
\text { for } 6 \text { weeks }\end{array}$ & $\begin{array}{c}\text { Standing Stork } \\
\text { Test }\end{array}$ & $\begin{array}{l}\text { Significant } \\
\text { improvement in } \\
\text { IG }\end{array}$ \\
\hline Eid et al. 2017 [20] & $\begin{array}{l}\text { Randomized } \\
\text { Controlled Trial }\end{array}$ & $\begin{array}{c}\text { IG-Conventional Therapy for } 45 \\
\text { min+ Isokinetic Training } \\
\text { Program for } 15 \text { minutes } \\
\text { CG-Conventional Physical } \\
\text { Therapy for } 1 \text { hour }\end{array}$ & $\begin{array}{l}3 \text { times/week } \\
\text { for } 12 \text { weeks }\end{array}$ & $\begin{array}{l}\text { Biodex Stability } \\
\text { System }\end{array}$ & $\begin{array}{l}\text { Both groups } \\
\text { improved } \\
\text { Significantly. } \\
\text { However, IG > } \\
\text { CG }\end{array}$ \\
\hline Ghafar et al. 2017 [21] & $\begin{array}{l}\text { Randomized } \\
\text { Controlled Trial }\end{array}$ & $\begin{array}{l}\mathrm{G}_{1-} \text { Traditional Physical Therapy } \\
\text { for } 30 \text { mins } \\
\mathrm{G}_{2}=\text { Wii based Program (Virtual } \\
\text { Reality) for } 30 \text { mins }\end{array}$ & $\begin{array}{l}3 \text { times/week } \\
\text { for } 8 \text { weeks }\end{array}$ & $\begin{array}{l}\text { Pediatric } \\
\text { Balance Scale } \\
\text { Timed Up and } \\
\text { Go test } \\
\text { Five-Times-Sit- } \\
\text { To-Stand Test }\end{array}$ & $\begin{array}{l}\text { Significant } \\
\text { improvement in } \\
\text { both groups. In } \\
\text { between Group } \\
\text { comparison, } \mathrm{G}_{2} \\
\text { improved more } \\
\text { than } \mathrm{G}_{1}\end{array}$ \\
\hline
\end{tabular}


(Table 2). Continued.

\begin{tabular}{|c|c|c|c|c|c|}
\hline Studies & Study Design & Intervention & Duration & $\begin{array}{l}\text { Measuring } \\
\text { Scale/Test }\end{array}$ & Result \\
\hline Reis et al. 2017 [22] & $\begin{array}{l}\text { Randomized } \\
\text { Controlled Trial }\end{array}$ & $\begin{array}{l}\text { IG- Virtual Reality Therapy + } \\
\text { Usual activities, } 16 \text { sessions of } \\
20 \text { minutes CG-ND }\end{array}$ & $\begin{array}{l}4 \text { times/week } \\
\text { for } 4 \text { weeks }\end{array}$ & $\begin{array}{c}\text { Pediatric } \\
\text { Balance Scale }\end{array}$ & $\begin{array}{l}\text { Statistically } \\
\text { significant } \\
\text { improvement in } \\
\text { IG }\end{array}$ \\
\hline Hamed et al. 2016 [23] & $\begin{array}{l}\text { Randomized } \\
\text { Controlled Trial }\end{array}$ & $\begin{array}{c}\text { IG- Balance Exercise program+ } \\
\text { swimming Training program } \\
\text { (Aquatic Therapy) } \\
\text { CG-Balance exercise Program }\end{array}$ & $\begin{array}{l}3 \text { times/week } \\
\text { for } 12 \text { weeks }\end{array}$ & $\begin{array}{l}\text { Balance Master } \\
\text { System }\end{array}$ & $\begin{array}{l}\text { Significant } \\
\text { improvement in } \\
\text { IG }\end{array}$ \\
\hline $\begin{array}{l}\text { Gutiérrez-Vilahú et al. } \\
\qquad 2016[24]\end{array}$ & $\begin{array}{l}\text { Randomized } \\
\text { Controlled Trial }\end{array}$ & $\begin{array}{l}\mathrm{G}_{1} \text { and } \mathrm{G}_{1} \text { received same } \\
\text { treatment warm-up activities (5- } \\
10 \mathrm{~min}) \text {, core strength and } \\
\text { ballet barre exercises (15 min), } \\
\text { proprioception exercises and } \\
\text { balance ( } 20 \text { min), choreography } \\
(20 \text { min), improvisational } \\
\text { exercises and image } \\
\text { recognition in a mirror ( } 15 \mathrm{~min}) \text {, } \\
\text { and relaxation ( } 5-10 \text { min) for } \\
\text { the total } 90 \text { minutes }\end{array}$ & $\begin{array}{l}2 \text { times/week } \\
\text { for } 18 \text { weeks }\end{array}$ & $\begin{array}{c}\text { Center of } \\
\text { Pressure (COP) } \\
\text { closed and open } \\
\text { eyes }\end{array}$ & $\begin{array}{l}\mathrm{G}_{1} \text { improved } \\
\text { some parameters } \\
\text { related to the use } \\
\text { of } \\
\text { visual input in } \\
\text { controlling COP }\end{array}$ \\
\hline Eid'2015 [25] & $\begin{array}{l}\text { Randomized } \\
\text { Controlled Trial }\end{array}$ & $\begin{array}{l}\text { IG-Physical Therapy program + } \\
\text { Whole-body Vibration training } \\
\text { for } 1 \text { hour }+5-10 \text { min } \\
\text { CG- Physical therapy program } \\
\text { for } 1 \text { hour }\end{array}$ & $\begin{array}{l}3 \text { times/week } \\
\text { for } 6 \text { months }\end{array}$ & $\begin{array}{l}\text { Biodex Stability } \\
\text { System }\end{array}$ & $\begin{array}{l}\text { Significant } \\
\text { improvement in } \\
\text { both groups } \\
\text { IG>CG }\end{array}$ \\
\hline Ghaeeni et al. 2015 [26] & $\begin{array}{l}\text { Randomized } \\
\text { Controlled Trial }\end{array}$ & $\begin{array}{c}\text { IG- Jeffrey's Core stability } \\
\text { Exercises for } 45-60 \text { minutes } \\
\text { CG-ND }\end{array}$ & $\begin{array}{l}3 \text { times/week } \\
\text { for } 8 \text { weeks }\end{array}$ & $\begin{array}{c}\text { Modified Stork } \\
\text { Test }\end{array}$ & $\begin{array}{l}\text { Significant } \\
\text { progression in } \\
\text { static balance } \\
\text { was observed in } \\
\text { IG }\end{array}$ \\
\hline Park et al. 2014 [27] & $\begin{array}{l}\text { Quasi- } \\
\text { experimental } \\
\text { Study }\end{array}$ & $\mathrm{G}_{1}$ - Wobble Board Training & Not mentioned & $\begin{array}{l}\text { Center of } \\
\text { pressure (COP) } \\
\text { with eyes open } \\
\quad \text { and closed }\end{array}$ & $\begin{array}{l}\text { Significant } \\
\text { improvement } \\
\text { observed }\end{array}$ \\
\hline Nejadsahebi 2013 [11] & $\begin{array}{l}\text { Randomized } \\
\text { Controlled Trial }\end{array}$ & $\begin{array}{l}\text { IG-Rebound Therapy for } 20 \\
\text { minutes } \\
\text { CG-ND }\end{array}$ & $\begin{array}{l}3 \text { times/week } \\
\text { for } 8 \text { weeks }\end{array}$ & $\begin{array}{l}\text { BERG Standard } \\
\text { Test } \\
\text { Timed Up and } \\
\text { Go }\end{array}$ & $\begin{array}{l}\text { Significant } \\
\text { improvement in } \\
\text { IG, Dynamic } \\
\text { balance }>\text { Static } \\
\text { balance }\end{array}$ \\
\hline Villarroya et al. 2013 [28] & $\begin{array}{l}\text { Randomized } \\
\text { Controlled Trial }\end{array}$ & $\begin{array}{l}\text { IG-Whole Body Vibration } \\
\text { CG-Squat Exercises }\end{array}$ & $\begin{array}{l}3 \text { times/week } \\
\text { for } 20 \text { weeks }\end{array}$ & $\begin{array}{l}\text { Postural } \\
\text { Parameters } \\
(\mathrm{COP})\end{array}$ & $\begin{array}{l}\text { IG had a positive } \\
\text { effect on balance } \\
\text { only under } \\
\text { specific } \\
\text { conditions }\end{array}$ \\
\hline
\end{tabular}

IG-Intervention Group; CG-Control Group; G-Group; ND-Not Mentioned; COP-Centre of Pressure.

choreography showed improvement in some parameters related to the use of visual input in controlling COP. The majority of studies were observed to provide the optimal duration of intervention, 3 times/week for 6,8 , or 12 weeks, while certain studies constitute of prolonged duration of $18-20$ weeks or 6 months for the notable effects. Among various standardized balance assessments, Biodex Balance System, Stork Test, Timed Up and Go Test, Pediatric Balance Scale, and Center of pressure (COP) were used by a number of studies. However, due to heterogeneity among the selected articles and their outcomes, measures meta-analysis could not be performed. The characteristics and descriptions of the studies are represented in Tables 1 and 2.

\section{Risk of Bias}

The risk of bias based on the author's judgment for each included trial was performed using Cochrane's Risk of Bias Tool, as shown in Table $\mathbf{3}$ and Figure $\mathbf{2}$.

\section{Selection Bias}

\section{Random Sequence Generation}

All trials demonstrated low risk except for three studies $[5,17,24]$ that represented a high risk. 
Table 3: Cochrane Summary for Risk of Bias $(n=18)$

\begin{tabular}{|c|c|c|c|c|c|c|}
\hline Studies & 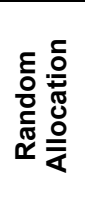 & 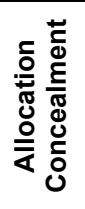 & 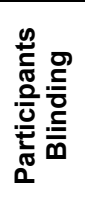 & 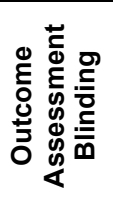 & 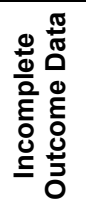 & 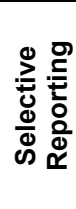 \\
\hline Alsakhawi et al. 2019 [15] & $\checkmark$ & $\checkmark$ & $x$ & $x$ & $x$ & $\checkmark$ \\
\hline Gheitasi et al. 2019 [16] & $\checkmark$ & $\checkmark$ & $x$ & $?$ & $\checkmark$ & $\checkmark$ \\
\hline Boer et al. 2019 [17] & $x$ & $x$ & $x$ & $x$ & $x$ & $\checkmark$ \\
\hline Akyol et al. 2018 [18] & $\checkmark$ & $\checkmark$ & $\times$ & $x$ & $\times$ & $\checkmark$ \\
\hline Kamatchi et al. 2018 [10] & $\checkmark$ & $\checkmark$ & $x$ & $x$ & $x$ & $\checkmark$ \\
\hline Carter et al. 2018 [5] & $x$ & $x$ & $x$ & $x$ & $x$ & $\checkmark$ \\
\hline Reis et al. 2017 [22] & $\checkmark$ & $\checkmark$ & $x$ & $x$ & $x$ & $\checkmark$ \\
\hline Hamed et al. 2016 [23] & $\checkmark$ & $\checkmark$ & $x$ & $x$ & $x$ & $\checkmark$ \\
\hline Gutiérrez-Vilahú et al. 2016 [24] & $x$ & $x$ & $\checkmark$ & $x$ & $\checkmark$ & $\checkmark$ \\
\hline Eid 2015 [25] & $\checkmark$ & $\checkmark$ & $x$ & $\checkmark$ & $\checkmark$ & $\checkmark$ \\
\hline Ghaeeni et al. 2015 [26] & $\checkmark$ & $\checkmark$ & $x$ & $x$ & $x$ & $\checkmark$ \\
\hline Park et al. 2014 [27] & $\checkmark$ & $\checkmark$ & $x$ & $x$ & $x$ & $\checkmark$ \\
\hline Nejadsahebi 2013 [11] & $\checkmark$ & $\checkmark$ & $x$ & $x$ & $x$ & $\checkmark$ \\
\hline Villarroya et al. 2013 [28] & $\checkmark$ & $\checkmark$ & $x$ & $x$ & $x$ & $\checkmark$ \\
\hline
\end{tabular}

$\checkmark$, indicates low risk of bias.

$\times$, indicates high risk of bias.

? , indicates that cannot ensure risk of bias.

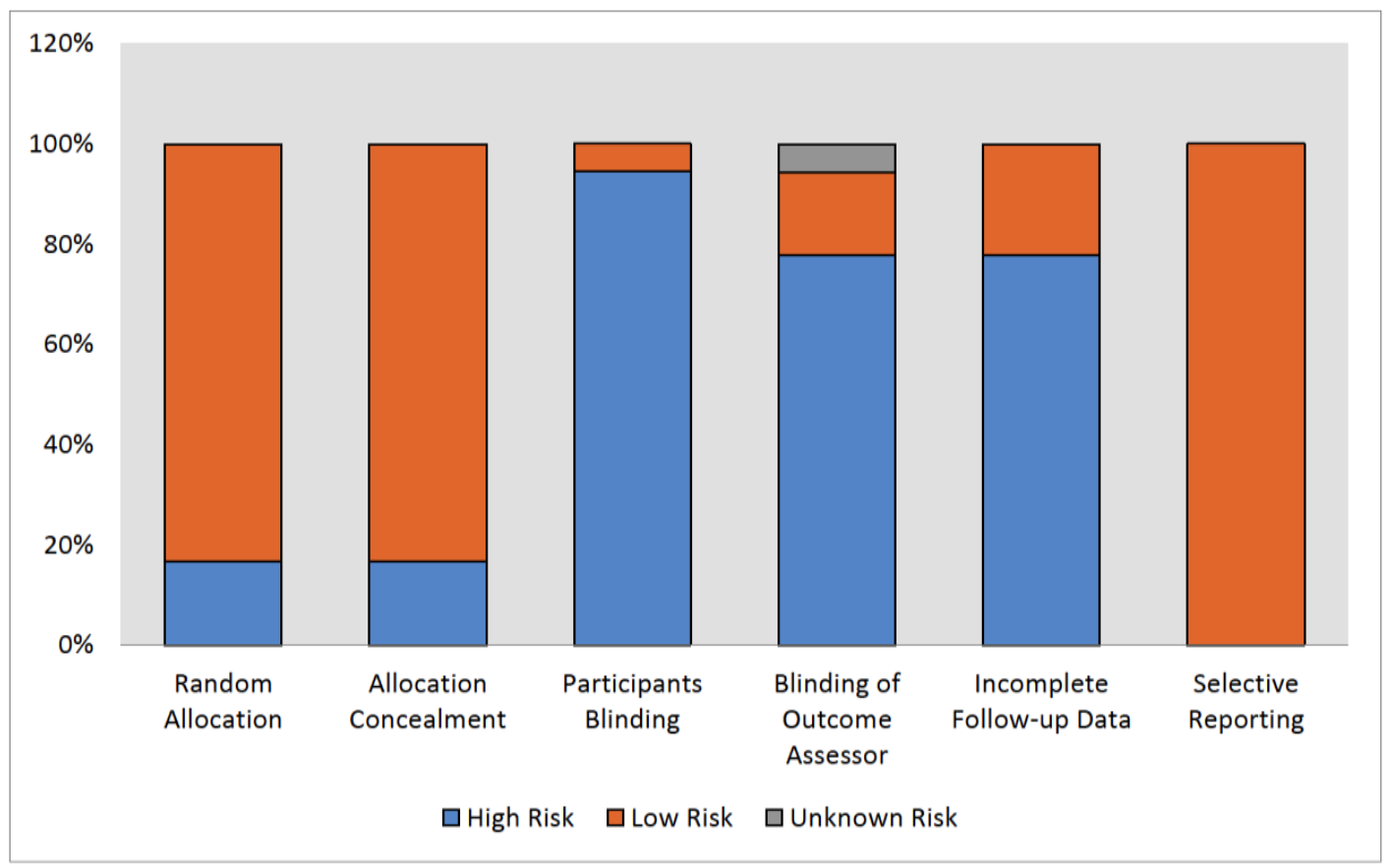

Figure 2: Represents Cochrane Risk of Bias Assessment. 


\section{Allocation Concealment}

Similarly, all included studies trials demonstrated low risk except for three studies $[5,17,24]$ that showed high risk.

\section{Performance Bias}

\section{Blinding of Participants and Personnel}

Only one study [24] out of eighteen showed a low risk of bias.

\section{Detection Bias}

\section{Blinding of Outcome Assessment}

Three studies $[20,21,25]$ reported a low risk of bias. However, one study [16] showed unknown risk while the remaining studies demonstrated high-risk bias [5, 10-12, 15, 17-19, 22-24, 26-28].

\section{Attrition Bias}

\section{Incomplete Outcome Data}

Four out of eighteen studies [16, 20, 24, 25] reported the loss to follow-up data and incomplete outcome data, while the remaining showed the high risk of bias [5, 10-12, 15, 17-19, 21-23, 26-28].

\section{Reporting Bias}

\section{Selective Reporting}

All the included studies showed a low risk of bias. $[5,10-12,15-28]$.

\section{DISCUSSION}

The review summarizes various interventions used to improve balance in persons with DS in the literature. The synthesized article into this commentary was reviewed by authors to ascertain the desired verdict.

\section{Core Stability Exercises}

Alsakhawi et al. [15] established the benefits of core stability training via Jeffrey's protocol included lumbarpelvic proprioception retraining, specific spinal stabilization exercises, muscle contractions, and abdominal maneuvers in combination with treadmill and traditional training. Both the treatment approaches were found to be equally effective in improving static and dynamic balance as well as gait patterns. Besides, Gheitasi et al. [16] used some games and recreational practices for the motivation of training that has led to a significant statistical improvement between training groups, in particular to static balance in the Swiss ball training group. Moreover, the increase was higher in the dynamic balance and muscle endurance in the TRX training group. Besides, Ghaeeni et al. [26] showed that core stability exercises improve the static balance of Down syndrome children by developing strength and endurance of the core stabilization area. The mechanism is most probably related to the integration of proprioceptive feedback due to closed chain movements.

\section{Virtual Reality}

Ghafar et al. [21] used VR based Wii training constituted of football heading game that improves movements of trunk and extremities, Ski Slalom that elicits balance strategies and loading of the lower limbs, Table Tilt game elicits control over the whole body through dynamic balance on a virtual balance board. Likewise, Reis et al. [22] used two games from Kinect Adventures $®$, i.e., River Rush $®$ and Hall of ricochets $®$ that leads to improved quality of motor responses and a higher balancing score statistically significant. The findings revealed that VR is superior to traditional physiotherapy training in improving the balance in DS children that is partially due to the training repetition that enhances the recovery of brain plasticity as well as motivates patients to interact and contribute in simulated environments to perform a specific therapeutic exercise.

\section{Aquatic Therapy}

Hamed et al. [23] established a statistically significant difference in balance scores due to the hydrostatic pressure of water that in turn reduces the static work, thereby counterbalance the gravity and supports the weight for the assumption of correct posture. Similarly, Boer et al. [17] found medium effect sizes for the functional ability and dynamic balance and small effect size for static balance. Besides, aquatic therapy was safer than other interventions as a child in water may exert greater freedom of movement that may assist in increasing the body's proprioception and making the movement easier to learn.

\section{Vestibular Stimulation}

Carter et al. [5] ascertain that vestibular stimulation exercise programs could increase balance and agility in the DS population, possibly increasing their functional ability. Similarly, Kamatchi et al. [10] also indicated the higher effectiveness of vestibular stimulation over 
weight-bearing exercises in improving balance; however, these exercises are more consistent with providing proprioceptive feedback to enhance movement control with respect to speed and timing. Moreover, weight-bearing exercises have been shown to have significant effects on balance and gait in patients with no or moderate cognitive/physical impairment.

\section{Whole Body Vibration}

Eid [25] demonstrated the combined effectiveness of whole-body vibration in improving balance and muscle strength of knee flexors and extensors. This speculated that vibration might be one of the strongest methods for stimulating proprioceptors attributed to improving balance. Besides, Villarroya et al. [28] indicated that whole-body vibration had positive effects on balance only under specific conditions, with altered visual and somatosensory input.

\section{Miscellaneous Interventions}

Several studies advocated different interventions that have resulted in substantially different yet significant outcomes. Park et al. [27] indicated that wobble board training enhances the proprioception that leads to the compensation of disturbed vision in DS adolescents. Nejadsahebi [11] revealed that rebound therapy is effective in enhancing static and dynamic balance in DS children. Consequently, the benefits of exercise in this population are more than improving physical health. Furthermore, Gutiérrez-Vilahú et al. [24] suggested that dance therapy improved some of the parameters related to visual input in controlling COP as young DS individuals had impaired COP control when eyes open and closed as compared to the normal population. Akyol et al. [18] determined the significant effects of gymnastic training with music on dynamic balance and coordination in the DS population. Also, Ahmadi et al. [19] described the feasibility of functional training as a safer form of exercise with no reported adverse consequences documented to date. In addition, Eid et al. [20] declared isokinetic exercises as an acceptable form of exercise that encourages the DS participants to become more active as individuals who are at high risk of physical inactivity consequences. Didehdar and Kharazinejad [12] indicated profound effects of sensorimotor integration in improving static and dynamic balance in comparison to usual activities. Moreover, sensorimotor integration skills may also provide these children with the necessary time to participate in the favorite games along with their peers. These diverse interventions yield positive effects on the balance; however, due to the heterogeneity of recent trends, the implications are arguable.

\section{LIMITATIONS}

The number of studies did not provide an adequate description of the intervention; moreover, in some of the evidence, the control group and severity of the disability were not demarcated. Yet, more male participants were explored than females in a number of trials. Consequently, our study had a small sample size due to the scarcity of literature in the included tenure. Moreover, the small sample size limited the generalizability of results due to heterogeneity of age and intellectual disability. Some interventions had a shorter duration with no follow-up, so the desirable effects were unable to sustain. The diversity of interventions also has some clinical implications due to the higher risk of chronic diseases in the DS population. The underlying mechanism of some is still ambiguous.

Furthermore, certain studies used field tests to evaluate the research variables. Although in some of the interventions, usual activities were not controlled, that may lead to biased outcomes. In addition, outcome assessors were often found to be unblinded to the given intervention.

\section{STRENGTHS}

To the best of author's knowledge, no review article documenting emerging trends to improve balance in the DS population has been conducted in the world. The study scrutinized clinical trials that profoundly affect balance, analyzed on the standardized checklists to reduce bias and produce reliable conclusions.

\section{FUTURE RECOMMENDATIONS}

Further trials are suggested to be conducted on the aforementioned interventions that encourage and open a wide range of research to establish the most efficient program for the DS population. Therefore, future clinical trials can extend the duration of management with a larger sample size to determine the intervention's long-term sustainability to increase the generalizability of health-related outcomes.

\section{CONCLUSION}

It was concluded that all the included trials demonstrated significantly profound effects in improving the static and dynamic balance of Down 
syndrome patients. Therefore, none of the interventions was declared as superior to another in terms of obtained results. Furthermore, these diverse interventions need to be investigated more for better understating and generalizability of outcomes. In addition, age and disability spectrum along with underdiagnosed co-occurring conditions should be considered for the management of the Down syndrome population in future studies.

\section{ACKNOWLEDGEMENTS}

The corresponding author would like to thank Ms. Zainab Meher Hasan (President, A.C.E.L.P) for her expert guidance and invaluable suggestions.

\section{CONFLICT OF INTEREST}

The authors reported no disclosure or competing interests.

\section{FUNDING}

Not applicable.

\section{ABBREVIATIONS}

$$
\begin{aligned}
& D S=\text { Down syndrome } \\
& \text { COP }=\text { Center of Pressure } \\
& T R X=\text { Total Resistance Exercise } \\
& V R=\text { Virtual Reality } \\
& N D=\text { Not Mentioned } \\
& I G \quad=\text { Intervention Group } \\
& C G=\text { Control Group } \\
& n \\
& I G_{1}=\text { Number } \\
& I_{2}=\text { Intervention Group } 1 \\
& G_{1}=\text { Group } 1 \\
& G_{2}=\text { Group } 2 \\
& C_{1}=\text { Control Group } 1 \\
& \mathrm{CG}_{2}=\text { Control Group } 2 \\
& \mathrm{SBE}_{1}=\text { Swiss ball exercise }
\end{aligned}
$$

\section{REFERENCES}

[1] Foley C, Killeen OG. Musculoskeletal anomalies in children with Down syndrome: an observational study. Arch Dis Child 2019; 104(5): 482-7.

https://doi.org/10.1136/archdischild-2018-315751

[2] Under-five mortality rate (per 1000 live births) (SDG 3.2.1) [Internet]. Who.int. 2020 [cited 3 October 2020]. Available from: https://www.who.int/data/maternal-newborn-childadolescent-ageing/indicator-explorer-new/mca/under-fivemortality-rate-(per-1000-live-birth).

[3] Azfar M, Khan I, Iqbal N, Khawar N, Abid K. Oral health of individuals with down syndrome in Karachi, Pakistan. J Pak Dent Assoc 2018; 27(04): 191. https://doi.org/10.25301/JPDA.274.190

[4] Lim PQ, Shields N, Nikolopoulos N, et al. The association of foot structure and footwear fit with disability in children and adolescents with Down syndrome. J Foot Ankle Res 2015; 8(1): 4 .

https://doi.org/10.1186/s13047-015-0062-0

] Carter K, Sunderman S, Burnett SW. The effect of vestibular stimulation exercises on balance, coordination, and agility in children with Down syndrome. J Psychiatry Neurosci 2018; 6(2): 28-32.

https://doi:10.11648/j.ajpn.20180602.11

[6] Weber A, Martin K. Efficacy of orthoses for children with hypotonia: a systematic review._Pediatr Phys Ther 2014; 26(1): 38-47.

https://doi:10.1097/PEP.0000000000000011

[7] Popa CE, Dobrescu T. The Effectiveness of therapeutic physical exercises in improving balance and coordination in children with Down syndrome. Romanian Journal for Multidimensional Education/Revista Romaneasca pentru Educatie Multidimensionala 2017; 9(3). https://doi.org/10.18662/rrem/2017.0903.06

[8] Ruiz-González L, Lucena-Antón D, Salazar A, Martín-Valero R, Moral-Munoz JA. Physical therapy in Down syndrome: systematic review and meta-analysis. J Intellect Disabil Res 2019; 63(8): 1041-67. https://doi.org/10.1111/jir.12606

[9] Arumugam A, Raja K, Venugopalan $M$, et al. Down syndrome-A narrative review with a focus on anatomical features. Clin Anat 2016; 29(5): 568-77.

https://doi.org/10.1002/ca.22672

[10] Kamatchi K, Balachandar V, Kaviraja N. Comparative Evaluation of Weight-Bearing Exercise and Vestibular Stimulation on Balance in Children with Down syndrome. Int J Pharma Med Sci 2018; 9(2): 227-234.

http://dx.doi.org/10.22376/ijpbs.2018.9.2.b227-234

[11] Nejadsahebi $N$, Sadeghi $H$, Nabavi SM. Effect and sustainability of 8-weeks rebound therapy exercise on dynamic and static balance of high school male students affected by down syndrome. Adv Environ Biol 2013; 4294-9. https://doi.org/10.1016/j.jns.2013.07.2008

Didehdar D, Kharazinejad A. The effect of balance activity on Down syndrome boys. Phy Hea Spt Med 2019; 2: 70-78. https://doi.org/10.36811/jphsm.2019.110012

Li C, Chen S, How YM, Zhang AL. Benefits of physical exercise intervention on fitness of individuals with Down syndrome: a systematic review of randomized-controlled trials. Int J Rehabil Res 2013; 36(3): 187-95. https://doi.org/10.1097/mrr.0b013e3283634e9c

[14] Higgins JP, Altman DG, Gøtzsche PC, et al. The Cochrane Collaboration's tool for assessing risk of bias in randomised trials. BMJ 2011; 343: d5928.

https://doi.org/10.1136/bmj.d5928

[15] Alsakhawi RS, Elshafey MA. Effect of core stability exercises and treadmill training on balance in children with Down 
syndrome: randomized controlled trial. Adv Ther 2019; 36(9): 2364-73.

https://doi.org/10.1007/s12325-019-01024-2

[16] Gheitasi M, Bayattork M, Miri H, Afshar H. Comparing the Effect of Suspended and non-suspended Core Stability Exercises on Static and Dynamic Balance and Muscular Endurance in Young Males With Down Syndrome. Physiotherapy - a specialized Journal of Physiotherapy 2019; 9(3) :153-60. http://ptj.uswr.ac.ir/article-1-394-en.html

[17] Boer $\mathrm{PH}$, de Beer $\mathrm{Z}$. The effect of aquatic exercises on the physical and functional fitness of adults with Down syndrome: A non-randomised controlled trial. J Intellect Disabil Res 2019; 63(12): 1453-63. https://doi.org/10.1111/jir.12687

[18] Akyol B, Pektas S. The Effects of gymnastics training combined with music in children with Autism Spectrum Disorder and Down syndrome. Int Educ Stud 2018; 11(11): 45-51. https://doi.org/10.5539/ies.v11n11p46

[19] Ahmadi N, Peyk F, Hovanloo F, Hemati Garekani S. Effect of functional strength training on gait kinematics, muscle strength and static balance of young adults with Down syndrome. Int j motor control learn 2018; 1(1): 1-0. http://ijmcl.com/article-1-50-en.html

[20] Eid MA, Aly SM, Huneif MA, Ismail DK. Effect of isokinetic training on muscle strength and postural balance in children with Down's syndrome. Int J Rehabil Res 2017; 40(2): 12733.

https://doi.org/10.1097/MRR.0000000000000218

[21] Ghafar MA, Abdelraouf OR. Effect of virtual reality versus traditional physical therapy on functional balance in children with down syndrome: A randomized comparative study. Int J Physiother Res 2017; 5(3): 2088-94. https://doi.org/10.16965/ijpr.2017.146
Reis JR, Neiva CM, Pessoa Filho DM, et al. Virtual Reality Therapy: motor coordination and balance analysis in children and teenagers with Down syndrome. Eur J Hum Mov 2017 Jul 19; 38:53-67.

[23] Hamed SA, Osama SA, Azab AS. Effect of aquatic program therapy on dynamic balance in Down's syndrome children. JMSCR 2016; 4(3): 9938-42.

https://doi.org/10.18535/jmscr/v4i3.59

[24] Gutiérrez-Vilahú L, Massó-Ortigosa N, Costa-Tutusaus L, Guerra-Balic M, Rey-Abella F. Effects of a dance program on static balance on a platform in young adults with Down syndrome. Adapt Phys Activ Q 2016; 33(3): 233-52. https://doi.org/10.1123/apaq.2015-0048

[25] Eid MA. Effect of whole-body vibration training on standing balance and muscle strength in children with Down syndrome. Am J Phys Med Rehabil 2015; 94(8): 633-43. https://doi.org/10.1097/phm.0000000000000224

[26] Ghaeeni S, Bahari Z, Khazaei AA. Effect of core stability training on static balance of the children with Down Syndrome. Physical Treatments-Specific Physical Therapy Journal 2015; 5(1): 49-54. http://ptj.uswr.ac.ir/article-1-232en.html

[27] Park TJ. The effects of wobble board training on the eyes open and closed static balance ability of adolescents with down syndrome. J Phys Ther Sci 2014; 26(4): 625-7. https://doi.org/10.1589/jpts.26.625

[28] Villarroya MA, González-Agüero A, Moros T, Gómez-Trullén $\mathrm{E}$, Casajús JA. Effects of whole body vibration training on balance in adolescents with and without Down syndrome. Res Dev Disabil 2013; 34(10): 3057-65. https://doi.org/10.1016/j.ridd.2013.06.015

https://doi.org/10.6000/2292-2598.2021.09.02.6

(C) 2021 Adeeb et al.; Licensee Lifescience Global.

This is an open access article licensed under the terms of the Creative Commons Attribution Non-Commercial License (http://creativecommons.org/licenses/by-nc/3.0/) which permits unrestricted, non-commercial use, distribution and reproduction in any medium, provided the work is properly cited. 